\title{
Statistical Study on Perceived JPEG Image Quality via MCL-JCI Dataset Construction and Analysis
}

\author{
Lina $\operatorname{Jin}^{a}$, Joe Yuchieh Lin $^{a}$, Sudeng Hu ${ }^{a}$, Haiqiang Wang ${ }^{a}$, Ping Wang $^{a}$, loannis Katsavounidis ${ }^{b}$ Anne Aaron $^{b}$ and C.-C. Jay Kuo ${ }^{a}$ \\ ${ }^{a}$ University of Southern California, Los Angeles, USA \\ ${ }^{b}$ Netflix, Los Gatos, USA
}

\begin{abstract}
The subjective test for compressed visual content is typically conducted by few experts called golden eyes. Here, we attempt to characterize the visual experience on JPEG-coded images of ordinary people statistically. To achieve this goal, a new image quality database, MCL-JCI, is constructed and introduced in this work. We explain the test procedure and conduct a preliminary analysis on test results. It is observed that people can only differentiate a finite number of quality levels and the perceived quality plot is a stair function of the coding bit rate. The relationship between the perceived quality plot and image content is discussed.
\end{abstract}

\section{Introduction}

Digital image/video plays an important role in our daily life. To facilitate storage and delivery of digital visual data, many compression algorithms, such as JPEG, MPEG-2, H.264 and HEVC, have been developed in the last 30 years. Although the meansquared-error (MSE) and the peak-signal-to-noise (PSNR) have been widely used to measure the distortion and quality of compressed visual data, respectively, they are not highly correlated to human visual experience. To address this shortcoming, the development of new quality indices has been an active research field in the last decade [1]. Perceptual image/video coding based on new quality indices has been investigated $[2,3]$, yet there is no convincing evidence that the replacement of PSNR with another quality index brings a clear advantage. It appears that the conventional rate-distortion coding framework has reached a plateau, and one may question whether there is any fundamental research left in the field of image/video coding.

To address the above challenging question, we would like to point out one fundamental issue associated with today's visual coding paradigm first. Without loss of generality, we use the popular JPEG image compression standard as an example. The quality factor (QF) in JPEG is used to control the quality of a coded image. The higher the QF value, the better the quality. Although one can choose QF from 1 to 100 with an increment of one to obtain 100 coded images, humans can only differentiate a small number of quality levels [4]. This study also shows that, in contrast with the conventional rate-distortion function, the perceived quality-QF curve is neither continuous nor convex. Rather, human-perceived quality is a stair function of the QF value (or, equivalently, the bit rate). This stair function contains a couple of jump points, which can be interpreted as the just noticeable difference (JND) position between two adjacent quality levels. The preliminary study conducted in [4] consisted of five JPEG-coded images and five H.264-coded video sequences evaluated by 20 subjects. On one hand, the number of perceived quality levels and the position of each JND point vary from person to person. On the other hand, although the number of test contents and subjects in [4] was small, the data set as a whole did provide a meaningful statistical trend.

The PSNR objective quality measure plays a dominant role in today's standardization efforts. Whenever there is an informal subjective test, it is conducted by a couple of experts called golden eyes. Since these experts have rich experience in capturing compression artifacts, they can spot subtle compression artifacts that are not visible to ordinary subjects. Such a practice corresponds to the worst-case analysis. It attempts to differentiate the most subtle differences that may only be perceived by golden eyes. In our opinion, it is at least equally interesting, if not more, to ask the following question: "What are the percentages of ordinary subjects who can differentiate the quality of the same content coded by different methods or by the same method but with different parameter settings?" It demands a large-scale statistical study on human-perceived image quality. Such investigation is expected to have a great impact on the future development of perceptual-based image/video coding standards.

To achieve the above objective, one essential step is to build a large-scale compressed visual dataset that consists of a wide variety of contents viewed by a sufficient number of subjects. The current work is a small step towards this huge effort. Specifically, we expand the size of JPEG-coded images from 5 to 50 and extend the number of test subjects from 20 to 30 in building the MCLJCI (an acronym for "Media Communications Lab - JND-based Coded Images") dataset. It is available to the public [5]. In this work, we will explain the test procedure, report test results, and conduct a preliminary analysis on the relationship between the stair quality-QF plot and the image content.

The rest of this paper is organized as follows. The construction of the MCL-JCI dataset is discussed in Section II . The derivation of the stair quality function (SQF) is discussion in Section III. Preliminary analysis of this dataset is conducted in Section IV. Finally, concluding remarks are given in Section V .

\section{MCL-JCI Dataset Construction}

In this section, the process of building a human-centric quality database for JPEG-coded images, called MCL-JCI, is presented. MCL-JCI contains 50 source (or uncompressed) images of resolution 1920x1080. Each source image is coded by the JPEG encoder [6] 100 times with the quality factor (QF) set rang- 


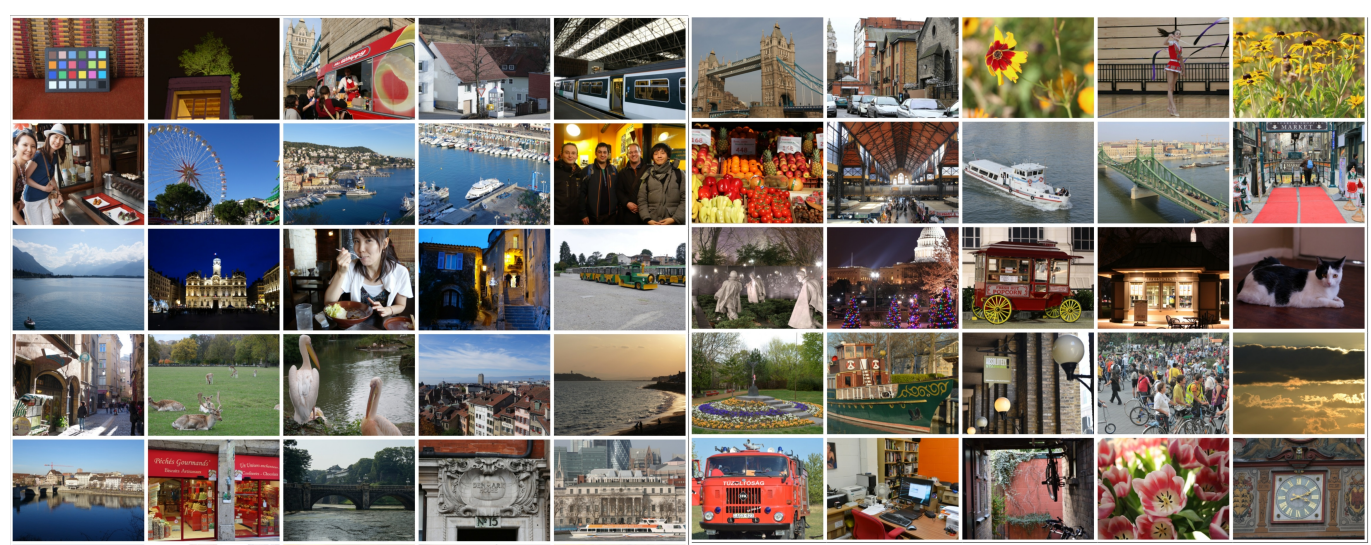

Figure 1: 50 source images in the MCL-JCI dataset.

ing from 1 to 100 . As a result, the whole MCL-JCI dataset consists of 5,050 images in total. More than 150 volunteers participated in the subjective test. The quality of coded images with respect to each source image is evaluated by 30 subjects by the bisection method introduced in [4]. As compared to other image quality databases (such as TID2013, TID2008, LIVE, CSIQ), MCL-JCI has the largest number of source and coded images for compressed image quality study.

\section{Dataset Design Objectives}

The MCL-JCI image quality dataset is constructed to answer the following questions.

- Q\#1: How many distinct quality levels can humans discern?

- $\mathrm{Q} \# 2$ : How to determine the boundaries between two adjacent quality levels?

- Q\#3: How to handle individual differences and obtain a single quality-versus-QF (or quality-versus-bit rate) function for the whole test group?

These questions are addressed in [4], and the answers to them are summarized below, respectively.

- A \#1: The number of distinguishable quality levels ranges from 3 to 8 for the middle 50th percentiles. It depends on the image content as well as each individual subject.

- A \#2: The boundaries between two adjacent quality levels are known as the JND points. Their location and height can be obtained by the bisection test methodology and analyzed using a post-processing procedure [7].

- A \#3: Although the subjective test results vary from person to person, we adopt a statistical method to integrate them into a single function.

Since the number of test contents and subjects in [4] was small, we will re-examine the above three questions by building a larger scale dataset, MCL-JCI, in this work.

\section{Image Content Selection}

To provide a good sampling of real world images, it is desired to build a dataset with a wide variety of content. The 50 source images included in MCL-JCI are shown in Fig. 1. Some of them were selected from the Ultra-eye database [8]. The diversity of this selection can be characterized by the spatial complexity index (also called the Spatial Information and denoted by SI), the colorfulness index (denoted by CF), and the semantic categories. The distribution of the spatial information and colorfulness of 50 source images in the MCL-JCI dataset is shown in Fig. 2. We

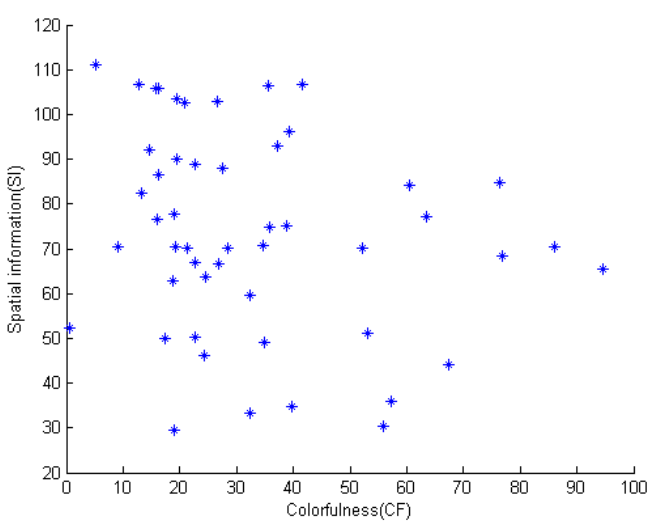

Figure 2: The distribution of spatial information and colorfulness of 50 source images in the MCL-JCI dataset.

see that the image set does have a wide coverage in this plot. For the definition of SI and CF, we refer to [9] and [10], respectively. Furthermore, the whole set of 50 source images can be classified into ten semantic or property-specific categories as given in Table 1. The nine semantic categories include: people, animals, plants, buildings, water or lake, sky, bridge, transportation vehicles (boats or cars) and indoor. The property-specific category is the dark scene.

Table 1: Ten semantic or property-specific categories and the number of source images in each category in the MCL-JCI dataset.

\begin{tabular}{|c|c|}
\hline & number \\
\hline People & 5 \\
\hline Animals & 3 \\
\hline Plants & 4 \\
\hline Buildings & 8 \\
\hline Water or Lake & 5 \\
\hline Sky & 3 \\
\hline Bridge & 3 \\
\hline Boats or Cars & 5 \\
\hline Indoor & 8 \\
\hline Dark Scene & 6 \\
\hline
\end{tabular}

Subjective Test Set-up and Procedure

More than 150 volunteers participated in the subjective test. 
They were equally stratified by gender and age (in the range from 20 to 40 years old). Ten of them were experts in the field of image quality assessment and/or image coding. The rest had little or no prior experience. They were seated in a controlled environment. The viewing distance was 2 meters (1.6 times the picture height) from the center of the monitor to the seat. The image pair was displayed on a 65 " TV with native resolution of $3840 \times 2160$. A subject compared two images displayed side by side and determined whether these two images are noticeably different. These two images are called the "anchor" and "comparison" images, respectively. The anchor image remains the same in the search of a specific JND point while the bisection method described in [4] is adopted to update the comparison image efficiently.

The JND point search is conducted in a sequential manner. In the first round, a subject finds the quality transition point between the best quality level and the second best quality level. This transitional point is called the first JND point. In this process, the anchor image is the image with $Q F=100$. Once the first JND point is determined, we use it as the anchor image to search for the 2nd JND point in the second round. Generally speaking, in the $n$th round, the image associated with the $(n-1)$ th JND point is used as the anchor image in the search of the $n$th JND point.

Given a QF interval whose right-end-point (i.e. the image of the largest QF value) is the anchor image, the goal of the bisection search algorithm is to determine the right-most image in the interval whose quality is noticeably different from that of the anchor image. The bisection algorithm for comparison image update is described below.

- Comparison Image Update. We choose the left-end-point of the interval as the first comparison image and ask each subject to tell whether there exists a noticeable difference between it and the anchor image. If no, no further search is needed for this interval since it does not contain a JND point. This happens when the anchor image is the last JND point. If yes, we select the image with the mid-QF value (rounded to the nearest integer) as the comparison image. If there exists a noticeable difference between it and the anchor image, we go to the middle of the right half interval. If no, we go to the middle of the left half interval. This procedure iterates until one of the following two termination cases is reached.

- Termination. There are two termination cases. Examples are shown in Figs. 3a and 3b, where ND and UD denote "noticeable difference" and "unnoticeable difference", respectively. For the first case, the process is terminated when the QF distance between the anchor image and the comparison image is equal to one. In other words, the $n$th JND point is the left neighbor of the $(n-1)$ th JND point. For the second case, a noticeable difference is observed at one comparison image (say, $Q F=a$ ) with respect to the anchor image but it cannot be observed at its right neighbor (say, $Q F=a+1$ ) as shown in Fig. 3b. Then, we choose $Q F=a$ as the next JND point.

We use Fig. 4 as an example to illustrate the sequential JND search process. We call the image with $Q F=100$ the 0 th JND point, and use it as the anchor image to determine the first JND, denoted by $Q F=x_{1}$, in the first round of search. A bisection search is conducted between QF interval $[1,100]$ to achieve this task. Then, we use the image with $Q F=x_{1}$ as the anchor image in the second round of search to find the 2nd JND image denoted by $Q F=x_{2}$. Then, the 2nd JND image is used as the anchor image in the 3 rd round of search. This process is repeated until all JND points are determined.

In the above discussion, the QF values of the best and worst quality images are set to $Q F=100$ and $Q F=1$, respectively. In practice, we can increase the worst $\mathrm{QF}$ to a slightly larger value that gives the lowest acceptable quality for a meaningful subjective test. This slight modification does not have any impact on the first several JND points since their anchor images have much higher QF values, and it was adopted in our subject test. Before the formal subjective test procedure, a small number of volunteers were asked to find the lowest acceptable QF parameter. For the selected QF values of the lowest acceptable quality images in the subject test, we refer to Fig. 5.

\section{Stair Quality Function (SQF)}

In this section, we explain a way to process the measured raw JND data and build an aggregate stair quality function (SQF) for each individual source image. The aggregate $\mathrm{SQF}$ is used to

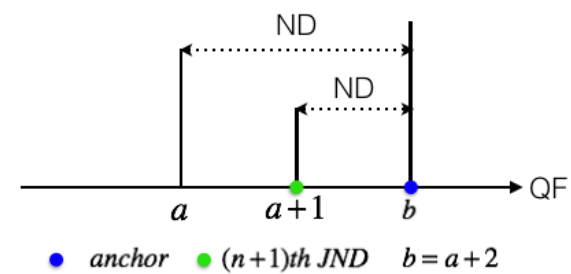

(a)

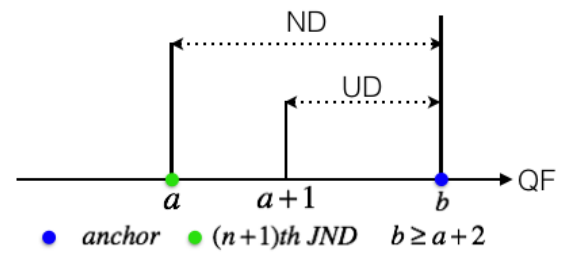

(b)

Figure 3: Illustration of two termination cases in the search of the $n$th JND point, $n=1,2, \cdots$, where integers $a$ and $a+1$ denote the QF values of two consecutive comparison points while integer $b$ is the QF value of the $(n-1)$ th JND point: (a) a noticeable difference is observed when the QF distance between the anchor image and the comparison image, $Q F=a+1$, is equal to one $(b=a+2)$, (b) a noticeable difference is observed at one comparison image $(Q F=a)$ but cannot be observed at its right neighbor $(Q F=a+1)$ with respect to the anchor image $b \geq a+2$.

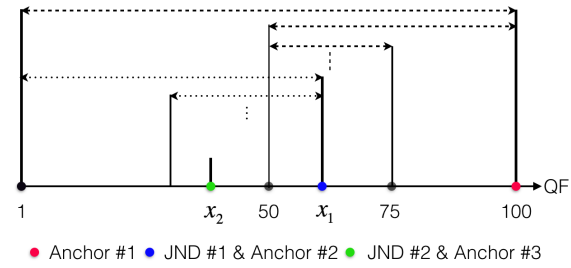

Figure 4: An example to illustrate of the sequential JND search process. 
characterize the mean experience of subjects in the test. Our discussion below is brief since the details can be found in [7]. It is included here for the sake of completeness.

Statistics of JND Numbers The average and the standard deviation (Std) of JND points for each source are given in Fig. 5. They are indicated by the blue bar and the green bar, respectively. The number of JND points does not vary a lot from one image to another. This is especially true by focusing on the interquartile range. Most of images have 4-7 JND points. The highest and lowest QF values for each image are also shown in Fig. 5. They are represented by the yellow curve and the orange curve, respectively. Knowing JND positions of compressed images is useful since it can provide more efficient compression service, such as providing same visual quality level with a lower number of bits.

\section{JND Position and Height Calculation}

We classify JND samples into three main groups according to their locations: high $\mathrm{QF}$, middle $\mathrm{QF}$ and low $\mathrm{QF}$ groups. The number of distinguishable quality levels and their JND positions depend on both image content and test subjects. Even for the same image and the same QF group, it is still difficult to group JND points since different subjects may have different numbers of JND points.

To proceed with statistical analysis, we need an underlying model for the JND distribution. We assume that the JND distribution is in the form of a Gaussian Mixture Model (GMM) with $N$ components. Mathematically, it can be expressed as

$$
f(x)=\sum_{i=1}^{N} \alpha_{i} \cdot \frac{1}{\sqrt{2 \pi} \sigma_{i}} \exp \left(-\frac{\left(x-q_{i}\right)^{2}}{2 \sigma_{i}^{2}}\right),
$$

where each component is a normal distribution with mean $q_{i}$ and variance $\sigma_{i}^{2}$, and $a_{i}$ is the mixture weight satisfying the constraint $\sum_{i=1}^{N} \alpha_{i}=1$. The set of parameters of GMM in Eq. (1); namely,

$$
\Theta=\left\{\alpha_{i}, q_{i}, \sigma_{i}\right\}, \quad i=1, \cdots, N,
$$

can be solved by the Expectation Maximization (EM) algorithm. The component number, $N$, of the GMM can be determined by the Bayesian information criterion (BIC), which helps strike a balance between data fitting performance and model complexity.

\section{From JND to SQF}

Once a GMM is built for each QF group (or region), the remaining task is to build the corresponding SQF. We first discuss the SQF for a single QF group. Let the normal function $N\left(x \mid q_{i}, \sigma_{i}\right)$ be the $i$ th Gaussian function in the GMM in the corresponding region. We associate the location of the $i$ th jump in the SQF with $q_{i}$ while its height is set to be proportional to the area under the weighted normal function $\alpha_{i} N\left(x \mid q_{i}, \sigma_{i}\right)$.

Next, we examine the SQF for all three QF regions combined together. The JND for the whole range can be obtained by combining the three JND sets. Mathematically, the JND function can be written as

$$
J N D(x)=\sum_{j=1}^{3} \sum_{i=1}^{N} H_{i j} \delta\left(x-q_{i j}\right)
$$

where $\delta(\cdot)$ is the Dirac delta function and $H_{i j}$ is the perceptual quality change degree at the $i$ th JND position in the $j$ th group (i.e. low, middle and high QF groups). The SQF is the normalized cumulative sum of JND function. Mathematically, it can be expressed as

$$
S Q F(x)=\frac{1}{\sum_{j=1}^{3} \sum_{i=1}^{N} H_{i j}} \int_{0}^{x} J N D(t) d(t),
$$

which is a monotonically increasing piecewise constant stair function.

Fig. 6 gives an example of the relationship between JND points and SQF. Raw JND samples were obtained from 30 subjects, histogram of all JND points and correspoing JND locations and height modeled by GMM were illustrated in Fig. 6 (b) and (c), respectively. Fig. 6 (d) gives the mean viewer experience by the SQFs, which serve as the ground truth of the observed JND points. The data of JND points for all 50 source images are given in the appendix.

\section{Relationship between Image Content and SQF}

In this section, we attempt to analyze the relationship between image content and its corresponding SQF. Generally speaking, image quality can be presented as a relation between produced and perceived quality [11]. The produced quality is the result obtained by a JPEG image codec. The perceived quality is related to the processing in the human visual system (HVS), which is a combination of early sensory processing and high-level cognitive processing. Image features extracted in the early sensory processing stage include: luminance, color, texture, etc. The cognitive processing stage involves individual knowledge, experience, expectation and emotion. In the following, we study the impact of the homogeneous region versus the textured region and the semantic object of three exemplary images on human perception.

The JPEG coding standard adopts block-based quantization of transform coefficients. The high frequency components are suppressed after JPEG compression. In textureless flat regions with gradual color change, the blocking artifact is visible to humans. In contrast, the distortion is less obvious in the textured region. For images with large smooth background (see Fig. 7(a) for an example), they tend to have more quality levels. We compare two images in different quality levels labeled by red and blue dots. Their QF values are 13 and 50, respectively. The quality of the image denoted by the blue dot is better than that of the image denoted by the red dot. We zoom into two regions (the sky and tree regions) to see the impact of texture on the quality level. The sky region has simple background while the tree region has complex texture. Visual quality degradation in the sky region is more visible than that in the tree region.

If an image is filled with rich texture, subjects tend to perceive few quality levels due to the spatial masking effect. One such example is given in Fig. 8, where the crowd appears in the form of random texture, which makes it difficult to observe blocking artifacts. In contract, the ground region has regular texture whose coding artifact is easier to observe.

Some images contain semantically meaningful regions that attract the attention of subjects and the compression artifact in these regions will be more visible than others. One example is shown in Fig. 9. The face region creates more quality levels than the cup region in the background. 


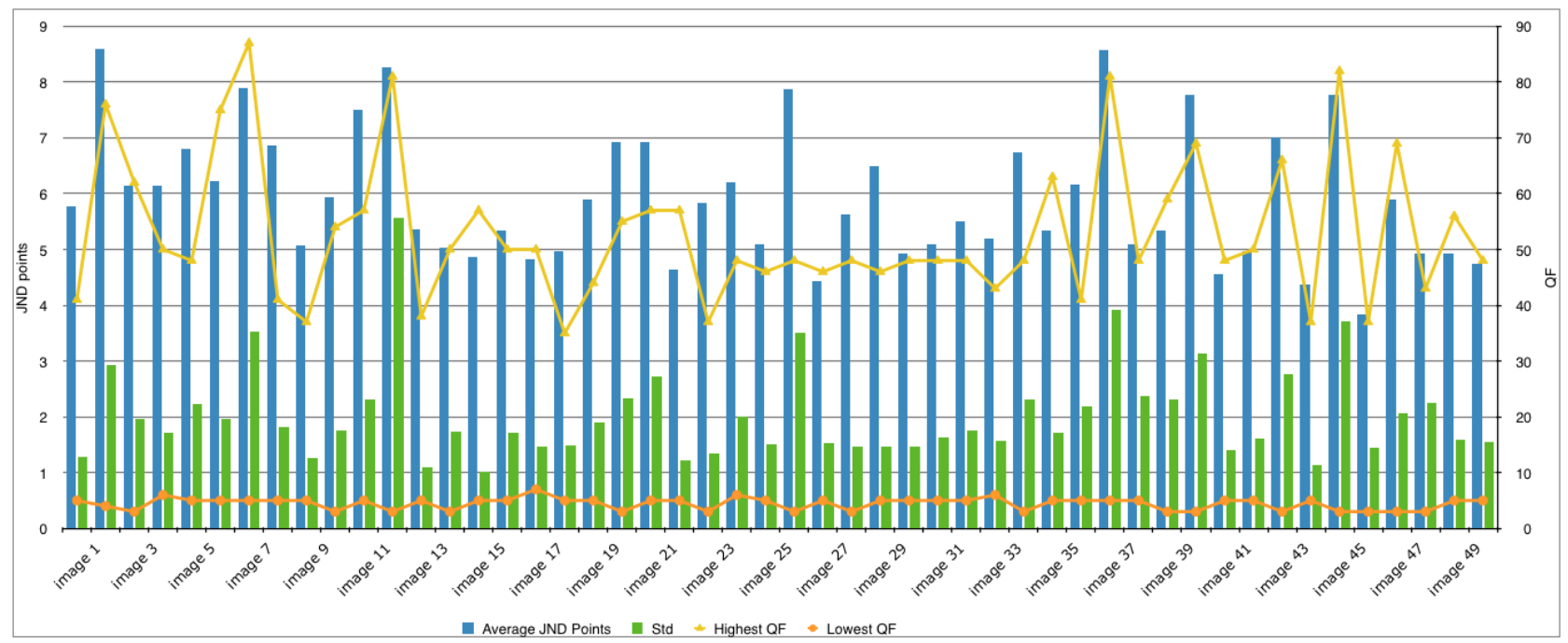

Figure 5: Statistics of the JND numbers and the highest and lowest QF values for 50 test images in MCL-JCI.

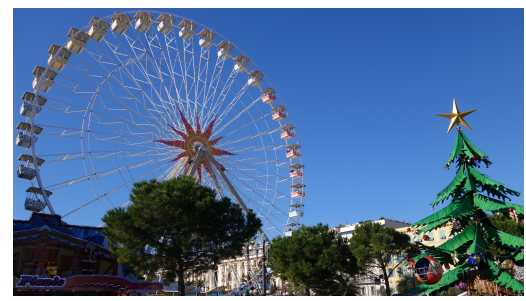

(a)

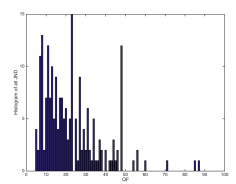

(b)

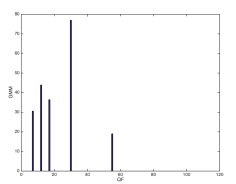

(c)

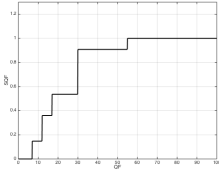

(d)
Figure 6: Illustration of the relationship between JND points and SQF: (a) Source image, (b) Histogram of all JND obtained form 30 samples, (c) JND location and height modeled by GMM (d) SQFs aggregating from GMM.

\section{Conclusion and Future Work}

A new methodology for human visual experience measurement was proposed in this work. A preliminary subjective test was carried out to collect the JND data to demonstrate the feasibility of this idea. The collected raw JND data was analyzed and post-processed to derive the stair quality function (SQF). The relationship between image content and SQF was discussed. We plan to use machine learning techniques to predict the SQF curves based on image content in the near future. We are also in the process of constructing a JND-based coded video quality dataset and will report the result soon.

\section{Appendix: The JND Table}

In the appendix, we provide the JND data for all 50 source images in Table 2. There exists a discontinuity at each JND point. The location of a JND point is its QF value while its height is defined to be the lowest position in the y-axis. The height of the boundary point with $Q F=100$ is normalized to unity, which de- fines the highest quality level. This point is called JND \#0, and the corresponding quality level is called the first quality level. Then, the first quality drops at the location of JND \#1 and its height defines the second quality level, and so on. This is illustrated in Fig. 10 .

\section{References}

[1] W. Lin and C.-C. J. Kuo, "Perceptual visual quality metrics: A survey," Journal of Visual Communication and Image Representation 22(4), pp. 297-312, 2011.

[2] S. Wang, A. Rehman, Z. Wang, S. Ma, and W. Gao, "Perceptual video coding based on ssim-inspired divisive normalization," Image Processing, IEEE Transactions on 22, pp. 1418-1429, April 2013.

[3] Y.-H. Huang, T.-S. Ou, P.-Y. Su, and H. Chen, "Perceptual ratedistortion optimization using structural similarity index as quality metric," Circuits and Systems for Video Technology, IEEE Transactions on 20, pp. 1614-1624, Nov 2010.

[4] J. Y.-C. Lin, L. Jin, S. Hu, I. Katsavounidis, Z. Li, A. M. Aaron, and C.-C. J. Kuo, "Experimental design and analysis of JND test on coded image/video," in SPIE Optical Engineering + Applications, pp. 95990Z-95990Z, International Society for Optics and Photonics, Aug 2015.

[5] "MCL-JCI Dataset." http://mcl.usc.edu/ mcl-jci-dataset/. Accessed: 2015-11-30.

[6] Independent JPEG Group, "JPEG image compression software." http://www.ijg.org. Accessed: 2015-03-20.

[7] S. Hu, H. Wang, and C.-C. J. Kuo, "A gmm-based stair quality model for human perceived jpeg images," arXiv preprint arXiv:1511.03398, 2015.

[8] H. Nemoto, P. Hanhart, P. Korshunov, and T. Ebrahimi, "Ultra-eye: Uhd and hd images eye tracking dataset," in Quality of Multimedia Experience (QoMEX), 2014 Sixth International Workshop on, pp. 39-40, Sept 2014.

[9] ITU, "Recommendation ITU-T P.910, Subjective video quality assessment methods for multimedia applications," International Telecommunication Union, Geneva, Switzerland 910, 1999.

[10] S. Winkler, "Analysis of public image and video databases for quality assessment," Selected Topics in Signal Processing, IEEE Journal 
of 6, pp. 616-625, Oct 2012.

[11] S. Jumisko-Pyykkö, V. Malamal Vadakital, and M. M. Hannuksela, "Acceptance Threshold: A Bidimensional Research Method for User-Oriented Quality Evaluation Studies."

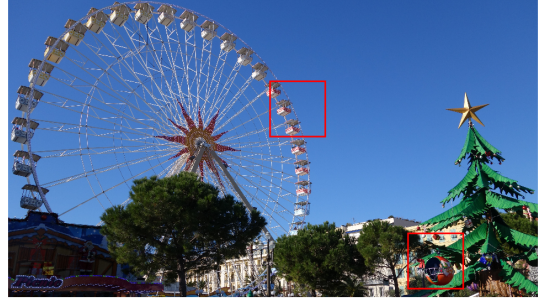

(a)

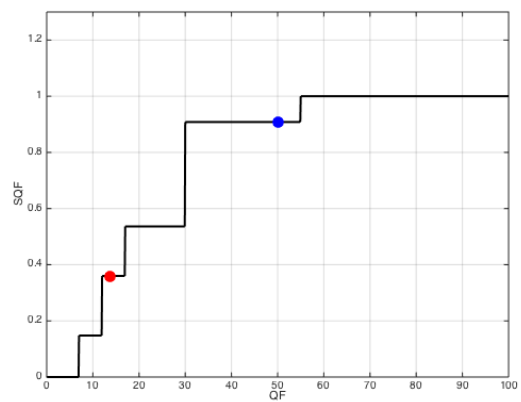

(b)

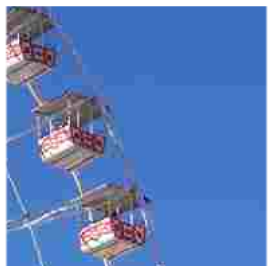

(c)

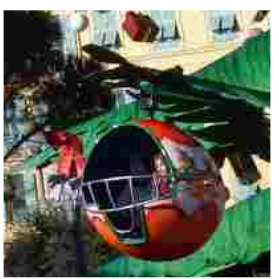

(e)

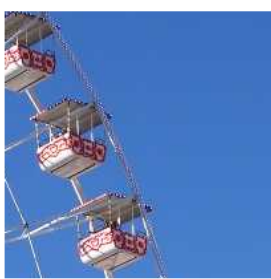

(d)

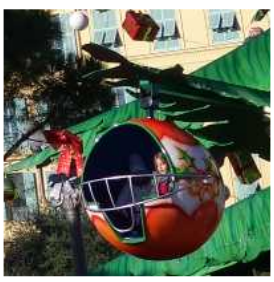

(f)
Figure 7: An exemplary image with a large homogeneous area (sky). (c, e) and (d, f) are regions from images denoted by red and blue dots in (b), respectively. 


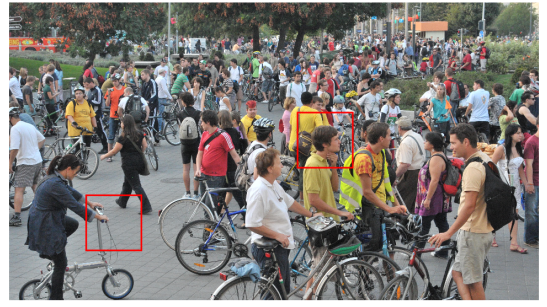

(a)

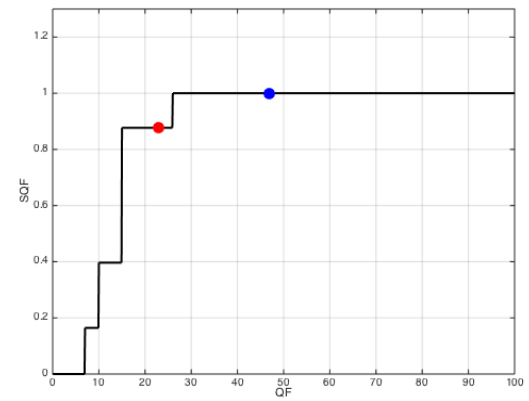

(b)

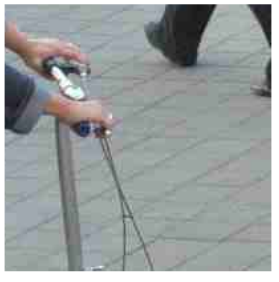

(c)

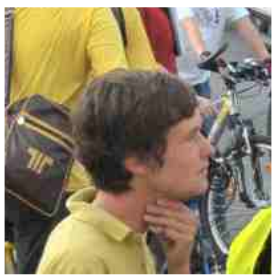

(e)

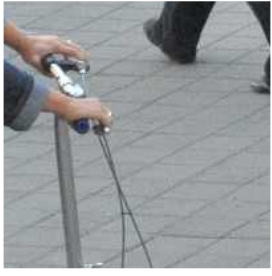

(d)

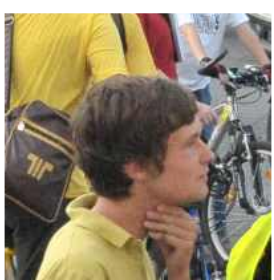

(f)
Figure 8: An exemplary image with complicated details (crowd), where (c) and (e) are regions from the coded image denoted by the red dot in (b) and (d) and (f) are regions from the coded image denoted by blue dot in (b), respectively.

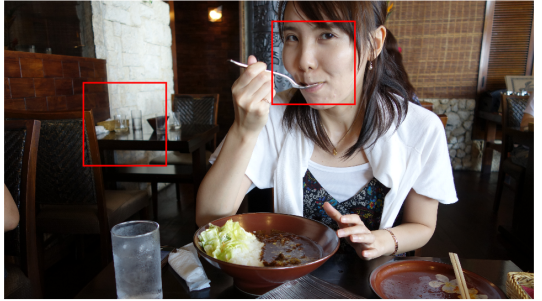

(a)

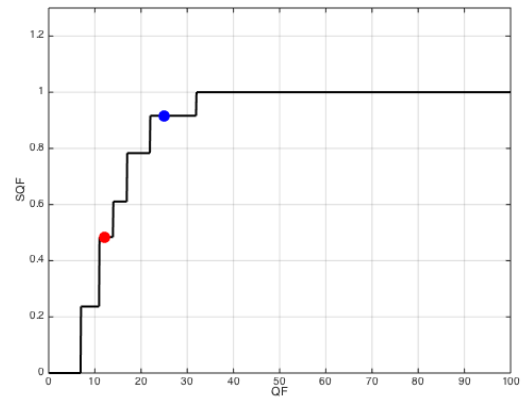

(b)

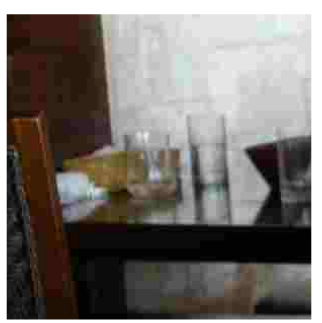

(c)

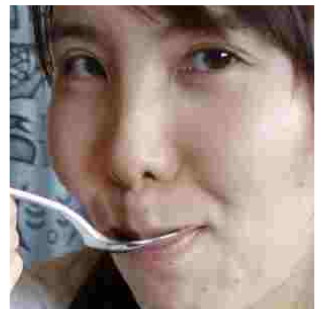

(e)

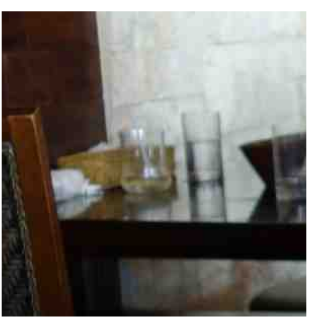

(d)

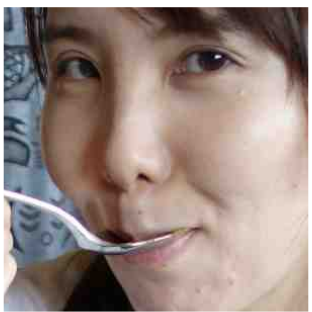

(f)
Figure 9: An exemplary image with a semantically meaningful object (face), where (c) and (e) are regions from the coded image denoted by the red dot in (b) and (d) and (f) are regions from the coded image denoted by blue dot in (b), respectively. 


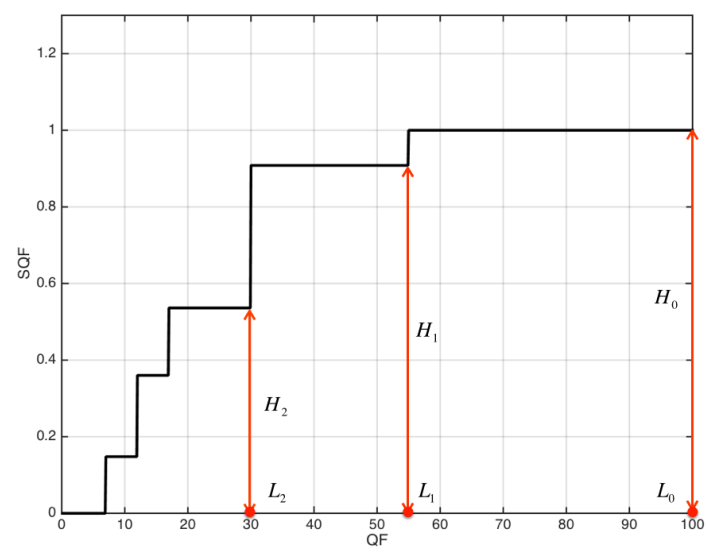

Figure 10: Illustration of JND locations and heights in Table 2 with the first 3 JND points for source image 07 as examples. 
Table 2: The list of JND locations and heights for 50 images in the MCL-JCI dataset [5], where $L$ stands for the location (i.e., QF value) and $H$ stands for the height (i.e. the relative perceptual quality) in the SQF plot, and JND \# 0 corresponds to the coded image with $Q F=100$.

\begin{tabular}{|c|c|c|c|c|c|c|c|c|c|c|c|c|c|c|c|c|}
\hline \multirow[b]{2}{*}{ Image id } & \multicolumn{2}{|c|}{ JND \# 0} & \multicolumn{2}{|c|}{ JND \# 1} & \multicolumn{2}{|c|}{ JND \# 2} & \multicolumn{2}{|c|}{ JND \# 3} & \multicolumn{2}{|c|}{ JND \# 4} & \multicolumn{2}{|c|}{ JND \# 5} & \multicolumn{2}{|c|}{ JND \# 6} & \multicolumn{2}{|c|}{ JND \# 7} \\
\hline & $\mathrm{L}$ & $\mathrm{H}$ & $\mathrm{L}$ & $\mathrm{H}$ & $\mathrm{L}$ & $\mathrm{H}$ & $\mathrm{L}$ & $\mathrm{H}$ & $\mathrm{L}$ & $\mathrm{H}$ & $\mathrm{L}$ & $\mathrm{H}$ & $\mathrm{L}$ & $\mathrm{H}$ & $\mathrm{L}$ & $\mathrm{H}$ \\
\hline 01 & 100 & 1.00 & 36 & 0.97 & 28 & 0.90 & 21 & 0.78 & 17 & 0.64 & 11 & 0.20 & & & & \\
\hline 02 & 100 & 1.00 & 47 & 0.92 & 31 & 0.80 & 26 & 0.70 & 21 & 0.50 & & & & & & \\
\hline 03 & 100 & 1.00 & 44 & 0.92 & 22 & 0.69 & 15 & 0.51 & 12 & 0.41 & 10 & 0.25 & & & & \\
\hline 04 & 100 & 1.00 & 40 & 0.94 & 28 & 0.92 & 21 & 0.72 & 16 & 0.59 & 13 & 0.37 & 10 & 0.29 & & \\
\hline 05 & 100 & 1.00 & 42 & 0.96 & 35 & 0.93 & 28 & 0.83 & 22 & 0.63 & 16 & 0.52 & 13 & 0.33 & 10 & 0.18 \\
\hline 06 & 100 & 1.00 & 51 & 0.98 & 33 & 0.91 & 20 & 0.57 & 12 & 0.27 & 8 & 0.15 & & & & \\
\hline 07 & 100 & 1.00 & 54 & 0.91 & 29 & 0.54 & 16 & 0.36 & 11 & 0.15 & & & & & & \\
\hline 08 & 100 & 1.00 & 32 & 0.90 & 21 & 0.65 & 16 & 0.49 & 13 & 0.30 & 10 & 0.19 & & & & \\
\hline 09 & 100 & 1.00 & 35 & 0.98 & 27 & 0.95 & 17 & 0.57 & 10 & 0.23 & & & & & & \\
\hline 10 & 100 & 1.00 & 35 & 0.94 & 24 & 0.90 & 22 & 0.83 & 19 & 0.71 & 16 & 0.61 & 13 & 0.37 & 10 & 0.23 \\
\hline 11 & 100 & 1.00 & 46 & 0.94 & 39 & 0.92 & 28 & 0.71 & 21 & 0.56 & 15 & 0.43 & 11 & 0.16 & & \\
\hline 12 & 100 & 1.00 & 68 & 0.92 & 53 & 0.80 & 39 & 0.65 & 28 & 0.53 & 15 & 0.18 & & & & \\
\hline 13 & 100 & 1.00 & 31 & 0.92 & 21 & 0.78 & 16 & 0.61 & 13 & 0.48 & 10 & 0.24 & & & & \\
\hline 14 & 100 & 1.00 & 35 & 0.92 & 22 & 0.69 & 16 & 0.56 & 11 & 0.31 & & & & & & \\
\hline 15 & 100 & 1.00 & 47 & 0.95 & 34 & 0.91 & 20 & 0.70 & 15 & 0.60 & 13 & 0.53 & 10 & 0.34 & 7 & 0.20 \\
\hline 16 & 100 & 1.00 & 45 & 0.95 & 32 & 0.92 & 18 & 0.52 & 10 & 0.29 & & & & & & \\
\hline 17 & 100 & 1.00 & 32 & 0.95 & 22 & 0.90 & 17 & 0.70 & 13 & 0.57 & 10 & 0.28 & & & & \\
\hline 18 & 100 & 1.00 & 30 & 0.93 & 22 & 0.85 & 19 & 0.75 & 16 & 0.64 & 14 & 0.54 & 10 & 0.26 & & \\
\hline 19 & 100 & 1.00 & 35 & 0.92 & 22 & 0.69 & 16 & 0.59 & 13 & 0.44 & 9 & 0.13 & & & & \\
\hline 20 & 100 & 1.00 & 46 & 0.88 & 31 & 0.65 & 22 & 0.56 & 15 & 0.31 & & & & & & \\
\hline 21 & 100 & 1.00 & 46 & 0.91 & 29 & 0.68 & 21 & 0.53 & 12 & 0.17 & & & & & & \\
\hline 22 & 100 & 1.00 & 42 & 0.90 & 18 & 0.55 & 10 & 0.27 & & & & & & & & \\
\hline 23 & 100 & 1.00 & 30 & 0.95 & 24 & 0.92 & 22 & 0.84 & 19 & 0.73 & 16 & 0.60 & 12 & 0.38 & 10 & 0.22 \\
\hline 24 & 100 & 1.00 & 31 & 0.92 & 22 & 0.77 & 17 & 0.56 & 11 & 0.19 & & & & & & \\
\hline 25 & 100 & 1.00 & 35 & 0.92 & 21 & 0.65 & 14 & 0.54 & 10 & 0.25 & & & & & & \\
\hline 26 & 100 & 1.00 & 45 & 0.92 & 30 & 0.66 & 21 & 0.51 & & & & & & & & \\
\hline 27 & 100 & 1.00 & 32 & 0.97 & 22 & 0.87 & 17 & 0.72 & 13 & 0.54 & 10 & 0.30 & & & & \\
\hline 28 & 100 & 1.00 & 43 & 0.94 & 33 & 0.85 & 22 & 0.72 & 19 & 0.59 & 11 & 0.16 & & & & \\
\hline 29 & 100 & 1.00 & 32 & 0.92 & 21 & 0.74 & 17 & 0.62 & 14 & 0.51 & 11 & 0.21 & & & & \\
\hline 30 & 100 & 1.00 & 33 & 0.91 & 21 & 0.67 & 16 & 0.56 & 11 & 0.17 & & & & & & \\
\hline 31 & 100 & 1.00 & 39 & 0.91 & 22 & 0.68 & 15 & 0.59 & 11 & 0.25 & & & & & & \\
\hline 32 & 100 & 1.00 & 35 & 0.91 & 22 & 0.78 & 18 & 0.67 & 15 & 0.52 & 11 & 0.31 & 7 & 0.16 & & \\
\hline 33 & 100 & 1.00 & 30 & 0.90 & 20 & 0.77 & 16 & 0.65 & 13 & 0.51 & 10 & 0.26 & 7 & 0.17 & & \\
\hline 34 & 100 & 1.00 & 39 & 0.91 & 23 & 0.60 & 15 & 0.47 & 11 & 0.20 & & & & & & \\
\hline 35 & 100 & 1.00 & 34 & 0.92 & 21 & 0.73 & 16 & 0.64 & 12 & 0.42 & 10 & 0.27 & 7 & 0.14 & & \\
\hline 36 & 100 & 1.00 & 29 & 0.89 & 22 & 0.79 & 19 & 0.71 & 16 & 0.60 & 11 & 0.18 & & & & \\
\hline 37 & 100 & 1.00 & 70 & 0.95 & 58 & 0.90 & 47 & 0.80 & 32 & 0.56 & 21 & 0.46 & 12 & 0.11 & & \\
\hline 38 & 100 & 1.00 & 34 & 0.92 & 21 & 0.70 & 15 & 0.56 & 10 & 0.25 & & & & & & \\
\hline 39 & 100 & 1.00 & 43 & 0.90 & 21 & 0.50 & 11 & 0.19 & & & & & & & & \\
\hline 40 & 100 & 1.00 & 50 & 0.93 & 32 & 0.69 & 21 & 0.53 & 13 & 0.25 & & & & & & \\
\hline 41 & 100 & 1.00 & 32 & 0.91 & 22 & 0.83 & 17 & 0.68 & 13 & 0.56 & 10 & 0.32 & & & & \\
\hline 42 & 100 & 1.00 & 31 & 0.92 & 22 & 0.79 & 16 & 0.60 & 12 & 0.44 & 10 & 0.28 & & & & \\
\hline 43 & 100 & 1.00 & 44 & 0.91 & 29 & 0.79 & 21 & 0.53 & 13 & 0.29 & & & & & & \\
\hline 44 & 100 & 1.00 & 25 & 0.88 & 14 & 0.40 & 9 & 0.16 & & & & & & & & \\
\hline 45 & 100 & 1.00 & 63 & 0.94 & 47 & 0.86 & 35 & 0.67 & 26 & 0.58 & 15 & 0.16 & & & & \\
\hline 46 & 100 & 1.00 & 28 & 0.92 & 20 & 0.77 & 13 & 0.59 & 10 & 0.36 & 7 & 0.15 & & & & \\
\hline 47 & 100 & 1.00 & 45 & 0.88 & 28 & 0.78 & 22 & 0.64 & 16 & 0.54 & 12 & 0.37 & & & & \\
\hline 48 & 100 & 1.00 & 41 & 0.98 & 33 & 0.92 & 23 & 0.75 & 17 & 0.62 & 14 & 0.52 & 10 & 0.28 & & \\
\hline 49 & 100 & 1.00 & 36 & 0.90 & 20 & 0.69 & 15 & 0.53 & 11 & 0.36 & 10 & 0.24 & & & & \\
\hline 50 & 100 & 1.00 & 31 & 0.94 & 20 & 0.81 & 15 & 0.69 & 12 & 0.44 & 9 & 0.27 & & & & \\
\hline
\end{tabular}

